\title{
Evaluating the effects of stress on asthma: a paradoxical challenge
}

\author{
M.D. Klinnert
}

Investigations into the relationship between emotional stress and asthma have a long and complicated history. Stemming from patient reports and clinician observations that asthma exacerbations were sometimes preceded by emotional reactions or severe stress, a series of empirical investigations was initiated in the 1960s. Study results were inconsistent, with some apparently methodologically rigorous studies showing relationships between emotional arousal and lung function changes, while others were unable to demonstrate such effects [1]. In the face of this confusing picture, a number of reviews have attempted to integrate data from the field, and in so doing have proposed pathways and mechanisms to explain how emotions or stress could affect asthma [1-4]. From the beginning, this area of research has been plagued by the paradox wherein the typical physiological reaction to emotional arousal was the opposite of physiological events associated with bronchoconstriction. The paper by LAUBE et al. [5] in the current issue of the European Respiratory Journal, which describes careful testing of a hypothesis generated by a theoretical model, represents another step forward in this tortuous process of investigation. Although the authors' hypothesis was not supported, the specifics of the unexpected results of the study provide another piece of the puzzle, and so contribute to a revision and refinement of the working model for investigating the interaction of stress and asthma.

The most important aspect of the results reported by LAUBE et al. [5] was the failure of the data to support the hypothesis; in fact, the results were the opposite of what was expected. The authors hypothesised that the pulmonary response to allergen exposure in individuals with allergic asthma would be potentiated by emotional arousal elicited by a stress interview. Instead, lung function testing showed less airway obstruction in response to allergens during stress visits than during nonstress, control visits. This suggested that the pulmonary response was attenuated rather than potentiated by the emotional stressor. Importantly, the results were consistent with one side of the paradox that has puzzled investigators from the beginning. It is well known (and is also documented in the study by LAUBE et al. [5]) that emotional arousal is accompanied by sympathetic activation, which is physiologically associated with bronchodilation. This contrasts with clinical observations and lore that have focused on emotions as precipitants of asthma attacks, which by their nature involve bronchoconstriction. In fact, in the study by LAUBE et al. [5], the results were consistent with the common sense view that emotional arousal should help to keep the airways open, rather than contribute to their constriction. Therefore, while the actual changes in pulmonary function may be clinically insignificant, the demonstration of statistically significant attenuation of effects under these conditions

Correspondence: M.D. Klinnert, National Jewish Medical and Research Center, 1400 Jackson Street, Denver, CO 80206, USA. Fax: 13032702141. E-mail: klinnertm@njc.org contributes to the big picture as scientists struggle to understand relevant variables and potential pathways involved in a complete model of the impact of stress on asthma.

To understand how these results help with the big picture, the particulars of the subjects and the conditions that allowed the demonstration of the attenuation effect must be identified. Simply defining what is meant by stress has been extremely problematic. Stress has been defined as demands or threats from an individual's environment that are appraised as taxing or exceeding one's ability to cope. Among the many possible challenging events people face, interpersonal difficulties or losses are consistently reported as having maximum impact. However, research in this area has often equated the effects of stress with those of emotional arousal [1]. There is a close relationship between the two, but they are not equivalent. The complexity of studying stress effects is illustrated in the LAUBE et al. [5] paper, where the subjects were asked by questionnaire about recent stressful events and were then were interviewed during the stress condition visit about the most severe of their reported stressful events. Although the study design relied on personally meaningful and disturbing recent events, the study manipulation involved the elicitation of emotional arousal. The subjects' experience of emotional arousal was corroborated by physiological indexes of sympathetic arousal. As pointed out by the authors in the discussion, the effects found here, while counter to the hypothesis, were probably directly related to the specific stressor used in the study, in this case emotional arousal.

Emotional arousal as a stress manipulation is to be distinguished from the effects of chronic stress on asthma. Chronic stress has been used to refer to challenges that have an impact on an individual for a long enough period of time that the body's physiological reactions have returned to baseline, although they may have instigated rebound or compensatory mechanisms in the process. The period of time referred to by the term "chronic" may be hours, days, weeks or years. Given time, the adjustments made by the body may result in a new homeostatic baseline, such as parasympathetic rebound in the short term or shifts in sympathetic/ parasympathetic balance in the long term [3], or increases in allostatic load [6]. The effects of chronic stress on asthma undoubtedly occur through different mechanisms than those mediating acute stress. There may well be an interaction between the two mechanisms, such that individuals with high levels of chronic stress have different pulmonary responses to acute emotional perturbations than individuals with low levels of chronic stress. It is intriguing to consider that the effect of life-long stress or adversity may be to alter those host characteristics associated with greater or lesser stress susceptibility. It is of interest, as pointed out by LAUBE et al. [5] in the discussion, that in this study the female reporting the highest level of stress was the one who failed to show a bronchodilation response to the manipulation. Among children with asthma, SANDBERG et al. [7] showed that severe negative life events increased the risk of children's 
asthma attacks over subsequent weeks, and that risk was magnified and brought forward in time if the child's life situation was characterised by multiple chronic stressors. SANDBERG et al. [7] interpreted these results in light of immunological changes and related viral infections that have been demonstrated to occur following negative life events and negative affect [8]. Whatever the mechanism, long-term stress (i.e. life-time adversity), recent life events and acute emotional arousal each probably have effects on asthma, and each probably has different effects depending on the stress histories of the individuals involved.

The likelihood of interactions between long-term or chronic stress and short-term or acute stressors underscores the necessity of defining the salient psychological and disease characteristics of the subjects that will determine how their asthma is impacted by stress. It is generally accepted that stress susceptibility varies across asthmatic individuals, as indeed it does among people without asthma. Historically, there has been some support for a distinction between intrinsic and extrinsic (or allergic) asthma; individuals with intrinsic asthma have been reported to be more vulnerable to emotional or stress-induced breathing problems [3]. It is also important to define the severity of subjects' asthma along multiple dimensions, such as symptom reports, medication requirements, pulmonary functions, presence of inflammation, etc. More challenging than defining disease characteristics is the definition of salient psychological characteristics, a challenge made particularly difficult by the fact that little is known about which psychological characteristics are salient. Studies linking psychological factors and asthma mortality and morbidity point to the importance of depression, histories of separation or loss, or presence of chronic conflict and relationship difficulties [9]. There is a need for research regarding the stress reactivity of individuals with histories of depression, trauma, relationship difficulties or chronic relationship conflict. BUSSE et al. [2] has suggested that individual differences in autonomic nervous system activity and reactivity may interact with stressors to produce different patterns of immunological and endocrinological changes. Only with such characterisation will it be possible to identify individuals or subgroups for whom stress effects may have a greater impact and only with such characterisation can we move closer to understanding which mechanism may be at play in specific types of asthmatics.

The study by LAUBE et al. [5] looks at one possible pathway among the numerous mechanisms posited to explain relationships between stress and asthma. As with all such studies, the findings require replication, hopefully with a larger number of subjects, more information about the subjects' psychological functioning and equally careful attention to methodology. That said, the study has made a valuable contribution by taking one possible pathway and exploring it carefully, with thorough documentation of the various relevant parameters. As in any area of science, when study subjects and procedural details of a study are carefully defined and described, other scientists can place the findings into the context of current knowledge, new hypotheses can be generated and a more complete knowledge of the phenomena can be realised.

\section{References}

1. Rietveld S, Everaerd W, Creer T. Stress-induced asthma: a review of research and potential mechanisms. Clin Exp Allergy 2000; 30: 1058-1066.

2. Busse WW, Kiekolt-Glaser JK, Coe C, Martin R, Weiss S, Parker S. Stress and asthma. Am J Respir Crit Care Med 1995; 151: 249-252.

3. Mrazek DA, Klinnert M. Psychoneuroimmunologic considerations. In: Ader R, Cohen $\mathrm{N}$, eds. Psychoneuroimmunology. Vol. 2nd Ed. San Diego, CA, Academic Press, 1991.

4. Wright RJ, Rodriguez M, Cohen S. Review of psychosocial stress and asthma: an integrated biopsychosocial approach. Thorax 1998; 53: 1066-1074.

5. Laube BL, Curbow BA, Fitzgerald ST, Spratt K. Early pulmonary response to allergen is attenuated during acute emotional stress in females with asthma. Eur Respir J 2003; 22: 613-618.

6. McEwen B, Seeman T. Protective and damaging effects of mediators of stress. Elaborating and testing the concepts of allostasis and allostatic load. Ann NY Acad Sci 1999; 896: $30-47$.

7. Sandberg S, Paton J, Ahola S, et al. The role of acute and chronic stress in asthma attacks in children. Lancet 2000; 356: 982-987.

8. Cohen S, Tyrrell D, AP S. Negative life events, perceived stress, negative affect, and susceptibility to the common cold. J Pers Soc Psychol 1993; 64: 131-140.

9. Strunk RC, Mrazek DA, Wolfson Fuhrmann GS, LaBrecque JF. Physiological and psychological characteristics associated with deaths from asthma in childhood: A casecontrolled study. J Am Med Assoc 1985; 254: 1193-1198. 